\title{
Substrate-mediated interaction and electron-induced diffusion of single lithium atoms on $\mathrm{Ag}(001)$
}

\author{
V. Simic-Milosevic, ${ }^{1, *}$ M. Heyde, ${ }^{1}$ N. Nilius,${ }^{1}$ M. Nowicki, ${ }^{2}$ H.-P. Rust,${ }^{1}$ and H.-J. Freund ${ }^{1}$ \\ ${ }^{1}$ Department of Chemical Physics, Fritz-Haber-Institut der Max-Planck-Gesellschaft, Faradayweg 4-6, D-14195 Berlin, Germany \\ ${ }^{2}$ Institute of Experimental Physics, University of Wroclaw, Plac Maxa Borna 9, PL-50-204 Wroclaw, Poland
}

(Received 14 December 2006; published 14 May 2007)

\begin{abstract}
The adsorption properties and the mechanism of induced diffusion of single $\mathrm{Li}$ atoms on $\mathrm{Ag}(001)$ have been investigated at $5 \mathrm{~K}$ by means of the low-temperature scanning-tunneling microscopy. On the basis of Li-Li pair distances, we have observed that there is no preferential adsorption site on $\mathrm{Ag}(001)$. Li adatoms form pairs with one preferred adatom distance, most likely due to long-range interaction between Li adatoms mediated by electrons from the bulk. The manipulation experiments show that diffusion of single Li adatoms can be induced upon electron transfer into an unoccupied electronic state connected to a single Li adatom. The existence of such Li-induced state is also confirmed by spectroscopic measurements.
\end{abstract}

DOI: 10.1103/PhysRevB.75.195416

PACS number(s): 68.37.Ef

\section{INTRODUCTION}

Alkali metals are efficient promoters in heterogeneous catalysis. ${ }^{1-3}$ Even small amounts of alkali-metal atoms adsorbed on noble or transition metals enhance the reactivity of a catalyst toward various reactions. It has been shown that dissociation of species such as $\mathrm{N}_{2}$ or $\mathrm{NO}$ on $\mathrm{Fe}(100)$ and $\mathrm{Rh}(100)$, as well as oxidation of $\mathrm{CO}$ on $\mathrm{Ni}(111)$, is promoted upon alkali adsorption. ${ }^{1-3}$ Additionally, the formation of alkali-induced quantum-well states, which may modify the surface reactivity, has been observed in $\mathrm{Na} / \mathrm{Cu}(111)$ and $\mathrm{Cs} / \mathrm{Cu}(111)$ systems. ${ }^{4-6}$ Thus, the detailed understanding of alkali-induced properties is necessary for facilitating the design of a new efficient catalyst. Furthermore, alkali-substrate systems are presently of interest for practical use in lithiumion batteries. Some rechargeable $\mathrm{Li}$-ion batteries have anodes that contain alloy compositions which include silicon, copper, and silver. In such systems, the adsorption and the diffusion of light alkali-metal ions at the anode material are of importance for the charging-recharging mechanism.

Alkali adsorption seems to be a simple example for chemisorption; however, its properties cannot be explained always with conventional pictures. Generally, the adsorption properties of single alkali-metal atoms on metal surfaces are explained with the Langmuir-Gurney model. ${ }^{7-13}$ This model proposes a very simple mechanism where the alkali atom transfers completely its valence $s$ electron to the substrate and adsorbs in a pure ionic $(+1)$ charge state. ${ }^{11}$ The main consequence of the proposed model is that the dominant interaction between alkali adatoms over a wide range of coverages is the repulsive dipole-dipole interaction. Since ionic bonds are not directional and commonly maximize coordination, at least at low coverage, alkali atoms should be adsorbed in high-coordinated hollow sites.

However, it has been shown for some alkali-substrate combinations, such as $\mathrm{Rb}$ on $\mathrm{Al}(111),{ }^{14} \mathrm{Cs}$ and $\mathrm{K}$ on $\mathrm{Cu}(111),{ }^{15}$ and $\mathrm{Li}$ on $\mathrm{Ru}(0001),{ }^{16}$ that alkali-metal atoms adsorb in low-coordinated sites. This is explained by the very small energy differences between the various adsorption sites for alkali adsorbates, caused by the loosely bound valence electron of the alkali-metal atom. ${ }^{17,18}$ Therefore, it is often not possible to predict the adsorption site for alkali-substrate systems. Additionally, for certain alkali-substrate combinations, such as $\mathrm{Na}, \mathrm{K}$, and $\mathrm{Rb}$ on $\mathrm{Al}(111)$, it was shown that at relatively low coverage, alkali-metal atoms aggregate into small islands at low temperature. ${ }^{19}$ Density-functional theory studies showed that such condensation occurs as the consequence of a balance between the adsorbate-adsorbate and adsorbate-substrate interactions. ${ }^{20}$ Lighter alkali-metal atoms induce weaker dipole moments upon adsorption and have higher cohesive energies compared to the heavier ones, and it should be expected that light alkali-metal atoms have larger tendency to condense. Additionally, it has been shown by low-energy electron-diffraction (LEED) experiments that if $\mathrm{K}$ adsorption on $\mathrm{Al}(111)$ is performed at $300 \mathrm{~K}$, a different process, namely, the surface adatom substitution, occurs. ${ }^{21}$

Concerning adsorption of light alkali-metal $\mathrm{Li}$ atoms on metal surfaces (Mo, Ru, W, Ir, Pt), it has been shown by nuclear-spin relaxation experiments that the chemical bond is not purely ionic. ${ }^{22}$ Furthermore, the substitutional adsorption for $\mathrm{Li}$ on $\mathrm{Al}(111)$ and the structure of the $\operatorname{Al}(111)-(\sqrt{3}$ $\times \sqrt{3}) R 30^{\circ}$-Li phase at room temperature have been determined by LEED. ${ }^{23}$ Similar results are obtained by LEED and scanning-tunneling microscopy (STM) for Li adsorption on $\mathrm{Cu}(001)$ at room temperature. It has been shown that depending on the coverage, different two-dimensional surface alloys are formed, which is manifested in a structural change from a $(2 \times 1)$ to $(3 \times 3)$ and $(4 \times 4)$ superstructures. ${ }^{24,25}$ Additionally, thermally induced diffusion of $\mathrm{Li}$ adatoms in a homogeneous alkali-metal adlayer on $\mathrm{Ru}(001)$ has been investigated with nuclear-magnetic-resonance techniques using radioactive isotope ${ }^{8} \mathrm{Li}$ as a probe. ${ }^{26}$

In this study, we investigate the adsorption properties and the mechanism of induced diffusion of $\mathrm{Li}$ atoms on $\mathrm{Ag}(001)$ by means of the STM. We show that even at low temperature, the condensation of $\mathrm{Li}$ atoms on $\operatorname{Ag}(001)$ occurs, resulting in the formation of $\mathrm{Li}$ adatom pairs with one preferred adatom distance. As an origin of the observed effect, we suggest a long-range interaction between $\mathrm{Li}$ adatoms on $\operatorname{Ag}(001)$ mediated by bulk electrons. The analysis of STM images shows that there is no preferential adsorption site on $\mathrm{Ag}(001)$ for $\mathrm{Li}$ adatoms. This indicates a small energy difference between the various adsorption sites for $\mathrm{Li}$ atoms on $\mathrm{Ag}(001)$. Finally, we show that nonthermal diffusion can be 
induced upon electron transfer into an unoccupied electronic state connected with a single $\mathrm{Li}$ adatom. This $\mathrm{Li}$-induced state is also resolved by spectroscopic measurements performed on single adatoms.

\section{EXPERIMENTAL PROCEDURE}

The experiments were performed with a custom-built, Eigler style, ultrahigh-vacuum STM operating at $5 \mathrm{~K}^{27}$ The microscope is equipped with a quartz tuning fork sensor for operation in both STM and atomic force microscopy mode. The $\operatorname{Ag}(001)$ sample was cleaned by repetitive cycles of $\mathrm{Ar}^{+}$ sputtering $(800 \mathrm{~V}, 10 \mathrm{~mA})$ and annealing at $700 \mathrm{~K}$. Lithium atoms were first deposited from a SAES alkali-metal dispenser at room temperature onto a transportable evaporator consisting of a tungsten filament. $\mathrm{Li}$ is then evaporated from the lithium-covered tungsten wire onto the $\operatorname{Ag}(001)$ sample directly in the STM at $8 \mathrm{~K}$. During spectroscopic measurements, the tunneling conductance spectra $d I / d V_{s}$ (the derivative of the tunneling current as a function of sample bias) were recorded while keeping the feedback loop on (maintaining a constant current during spectroscopy), which allow insights into the electronic structure of the sample.

\section{RESULTS AND DISCUSSION}

Figure 1(a) shows a STM image of the $\operatorname{Ag}(001)$ surface after deposition of about 0.01 monolayer (ML) lithium. Single Li adatoms appear at $V_{S}=300 \mathrm{mV}$ sample bias as $45 \mathrm{pm}$ high protrusions with a full width at half maximum (FWHM) of $0.6 \mathrm{~nm}$ [Fig. 1(b)]. The ellipsoidal features with an apparent height of $80 \mathrm{pm}$ and a FWHM of $1 \mathrm{~nm}$ at $300 \mathrm{mV}$ are dimers [Fig. 1(b)]. This is verified by the controlled separation of the two dimer atoms by applying voltage pulses with $3 \mathrm{eV}$ energy for a few seconds to these protrusions [Fig. 1(b)]. The Li adatoms show a tendency to aggregate into pairs. The distance distribution of $\mathrm{Li}$ adatom pairs shows a maximum in the range between 0.7 and $0.9 \mathrm{~nm}$ [Fig. 1(c)]. In the distance distribution, we count only $\mathrm{Li}$ pairs with separation not larger than $2 \mathrm{~nm}$. Distances smaller than $0.45 \mathrm{~nm}$ are not observed and most distances are larger than $0.6 \mathrm{~nm} .{ }^{28}$ This shows that a short-range repulsion influences the distribution of the atoms.

The adsorption sites of $\mathrm{Li}$ adatoms on $\operatorname{Ag}(001)$ are deduced from STM images. We compared distances measured between $\mathrm{Li}$ atoms that form a pair with the distances of potential adsorption sites of $\mathrm{Li}$ atoms on the $\mathrm{Ag}(001)$ lattice. In this procedure, both the crystal orientation and the relative orientation of Li pairs to the substrate are taken into account. On this empiric level, the structure models shown in Fig. $1(\mathrm{~d})$ are in good agreement with the experimental results. We find that $\mathrm{Li}$ binds to inequivalent sites and there is no preferential adsorption site for $\mathrm{Li}$ atoms on $\mathrm{Ag}(001)$.

In the following, we elucidate the mechanism behind the formation of Li adatom pairs. Generally, the interactions between adatoms depend significantly on their distance. Shortrange interactions are element specific and dominate at small separation, where electronic interactions lead to the formation of localized chemical bonds. ${ }^{29}$ According to the
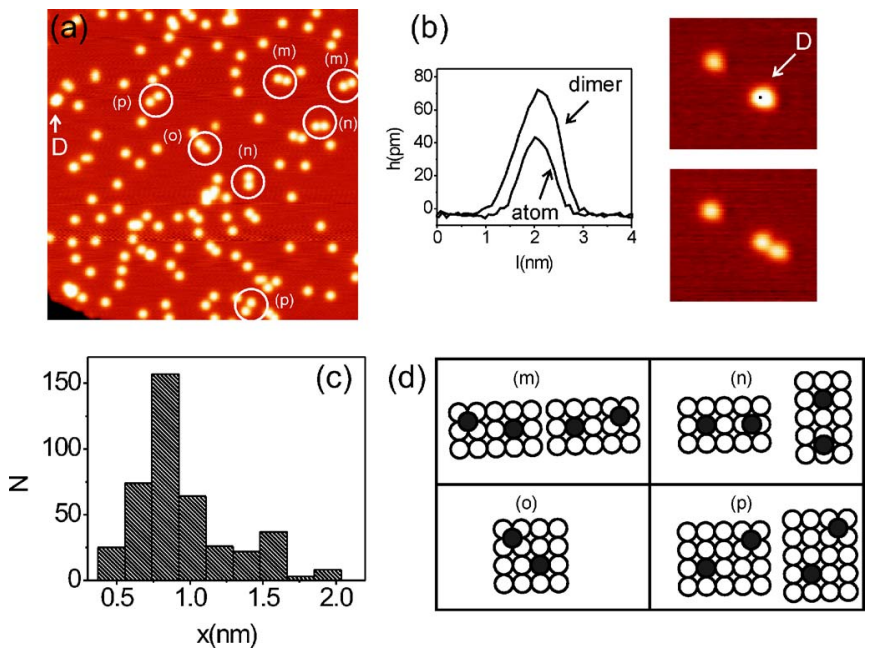

FIG. 1. (Color online) (a) Large-scale STM image showing the $\mathrm{Ag}(001)$ surface covered with Li adatoms (circular protrusions) and Li dimers (ellipsoidal protrusions, one is marked with $\mathrm{D}$ ). The coverage is approximately $1 \% \mathrm{ML}$, image size is $30 \times 30 \mathrm{~nm}^{2}$, and tunnel parameters are $V_{s}=300 \mathrm{mV}$ and $I=50 \mathrm{pA}$. Li adatoms form pairs (some surrounded by circles). For the marked Li adatom pairs, the models in (d) show potential adsorption sites on the $\operatorname{Ag}(001)$ lattice [for (m), distance $d$ is $0.768 \mathrm{~nm}$; for (n), $d$ is $0.722 \mathrm{~nm}$; for (o), $d$ is $0.672 \mathrm{~nm}$; for (p), $d$ is $0.896 \mathrm{~nm}$ ). (b) Line scans are measured above an adatom and a dimer (marked with D) in the STM image shown above on the right-hand side; image size is 8.1 $\times 7.2 \mathrm{~nm}^{2}$ and tunnel parameters are $V_{s}=300 \mathrm{mV}$ and $I=50 \mathrm{pA}$. A voltage pulse with $3 \mathrm{eV}$ energy was applied for $1.8 \mathrm{~s}$ to the ellipsoidal protrusion in the position marked by the black dot. After this procedure, two single $\mathrm{Li}$ atoms are observed instead of the dimer (the second STM image on the right-hand side). (c) Histogram of distances $d$ between adatoms for $30 \times 30 \mathrm{~nm}^{2}$ image size measured in 14 images.

Langmuir-Gurney model, Li atoms are positively charged and the dominant short-range interaction between $\mathrm{Li}$ ions should be repulsive. Consequently, small separations between $\mathrm{Li}$ ions should be excluded and only structures with the largest possible interatomic distances should be created.

At larger separations, long-range interactions between adatoms can be mediated by substrate electrons (Friedel-type interaction). The substrate electrons screen the potential induced by an adatom on the surface via charge-density oscillations (Friedel oscillations). The wave vector of these oscillations is $2 k_{f}$ since mostly electrons at the Fermi energy participate in the screening. These electrons may originate either from surface or bulk states, as shown in a recent STM experiment on $\mathrm{Ag}(110) .{ }^{30}$ Comparing to surface states, bulk states have significantly larger Fermi vectors [for $\operatorname{Ag}(111)$, $k_{f, s}=0.083 \AA^{-1}$ (Ref. 31) and $k_{f, b}=1.2 \AA^{-1}$ (Ref. 32)] and produce, therefore, Friedel oscillations with a significantly smaller wavelength. ${ }^{33}$ The long-range interaction decays asymptotically as $\cos \left(2 k_{f r}\right) / d^{2}$ in the presence of the partially filled surface-state band. ${ }^{34}$ In its absence, the interaction is bulk-state mediated and falls off more rapidly with $\cos \left(2 k_{f r}\right) / d^{5}{ }^{34}$ The adsorbates can interact via Friedel oscillations because the binding energy of one adsorbate depends on the substrate electron density, which, in turn, is perturbed 
(a)

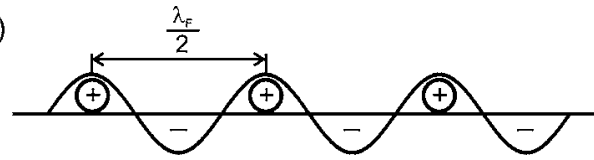

(b)

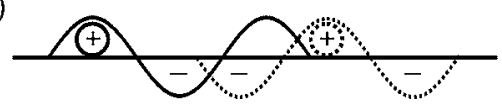

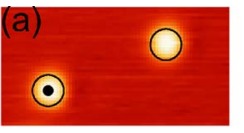

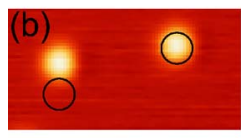

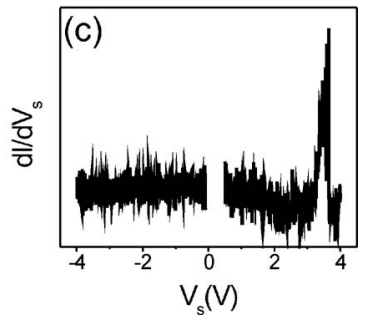

FIG. 3. (Color online) STM images show the same surface area (a) before and (b) after spectroscopic measurements; image size is $7.6 \times 3.8 \mathrm{~nm}^{2}$ and tunnel parameters are $V_{s}=300 \mathrm{mV}$ and $I$ $=50 \mathrm{pA}$. During the measurements, the tip was positioned above a single atom in the position marked by the black dot. (c) Two $d I / d V_{s}$ spectra were taken: first, in the region of occupied states between -0.1 and $-4 \mathrm{eV}$, and second, in the region of unoccupied states between 0.3 and $4 \mathrm{eV}$. $d I / d V_{s}$ spectra show no feature between -0.1 and $-4 \mathrm{eV}$. The position of the atom has not changed upon probing the occupied states. The probing of unoccupied states induces the diffusion of the $\mathrm{Li}$ atom. The sharp decrease in the $d I / d V_{s}$ spectra in the region of unoccupied states at about $3.6 \mathrm{eV}$ is a consequence of the induced diffusion.

trum was taken above the same atom in the region of unoccupied states (between 0.3 and $4 \mathrm{eV}$ ). There is a sharp increase in signal which could signify the presence of an unoccupied state connected to $\mathrm{Li}$ atoms with an onset above $3 \mathrm{eV}$. The following collapse of the signal at $3.6 \mathrm{eV}$ in Fig. 3(c) is due to the hopping of a Li atom away from the STM tip. This is verified by the STM image in Fig. 3(b) which clearly shows the position change of the Li atom at the lefthand side. The described behavior was observed for all $\mathrm{Li}$ atoms in our experiments. However, neighboring atoms were never affected by these measurements, manifesting the local character of the induced diffusion process.

In the following, we investigate the mechanism of this induced diffusion of single Li adatoms. We performed a series of manipulation experiments by following the same procedure as described below. The adatoms are first imaged with low bias and current [Fig. 4(a)]. Then, the tip is positioned above a particular atom [the position is marked by the black dot in Fig. 4(a)]. With the feedback loop on, the bias voltage is increased to the manipulation value and held unchanged for several seconds. Simultaneously, the $z$ signal is recorded
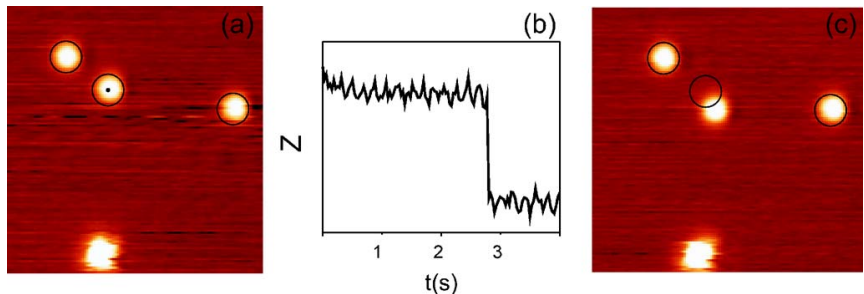

FIG. 4. (Color online) STM images (a) before and (c) after manipulation; image size is $12.5 \times 12.5 \mathrm{~nm}^{2}$ and tunnel parameters are $V_{s}=300 \mathrm{mV}$ and $I=50 \mathrm{pA}$. The electrons with an energy of $3.3 \mathrm{eV}$ are dosed for $4 \mathrm{~s}$ to the single atom in the position marked by the black dot. The circles show the positions of $\mathrm{Li}$ atoms before manipulation. (b) $z$ signal was recorded during the manipulation procedure. 

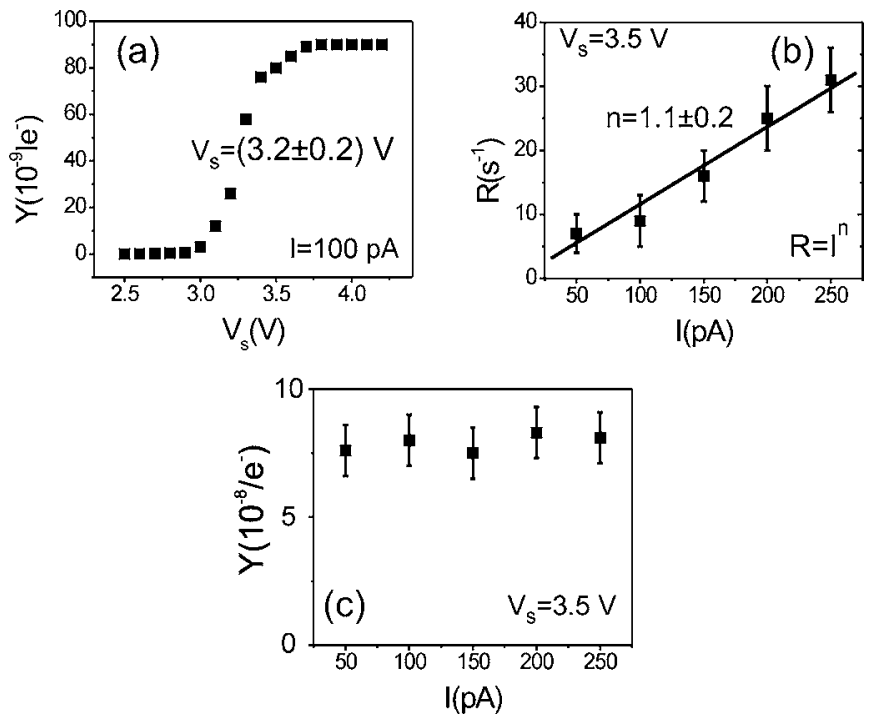

FIG. 5. (a) Yield $Y$ as a function of bias voltage $V_{s}$ for the induced diffusion. (b) Dependence of the reaction rate on the tunneling current. The solid line is a linear fit to the data corresponding to the power law $R=I^{n}$, with $n=1.1 \pm 0.2$, indicating that the diffusion is caused by a one-electron process. (c) Dependence of the yield on tunneling current.

[Fig. 4(b)]. The change in the $z$ signal manifests the success of the manipulation. The induced motion of the adatom is proven additionally by imaging the same area after the manipulation [Fig. 4(c)]. We systematically measured the hopping probability by repeating the experiment for different manipulation parameters. The voltage was varied between 2.5 and $4.5 \mathrm{~V}$, current between 50 and $250 \mathrm{pA}$, and manipulation time between 1 and $120 \mathrm{~s}$. From the $z(t)$ curves, it is possible to measure the time that an adatom spends in its initial position as a function of bias voltage $(2.5$ and $4.5 \mathrm{~V})$ and tunneling current (50 and $250 \mathrm{pA}$ ) and to calculate the number of injected electrons until the hopping event. The inverse of this number gives the yield per incident electron for each event [Fig. 5(a)]. From the dependence of the manipulation yield on the voltage, we determined the threshold for the induced diffusion to be at $3.2 \mathrm{eV}$. For a process induced by the tunneling electrons, the dependence of the rate $R$ on tunneling current $I$ is related to a power law $R=I^{n}$ with $n=1$ in a case of a single-electron event [Fig. 5(b)].11,42 The fit to the measured data gives a coefficient $n=1.1$, identifying the phenomenon as a one-electron process.

Our experiments were performed at elevated voltages and it is therefore difficult to differentiate between a fieldinduced mechanism or an electron-induced process for the hopping. To elucidate the correct mechanism, we calculated the hopping yield for particular tunneling currents (between 50 and $250 \mathrm{pA}$ ). For the approximation, we assumed a typical tunneling gap distance of approximately $8 \AA$, a tunneling resistance of $0.1 \mathrm{G} \Omega$, and a decrease in tip-sample separation by $1 \AA$ if tunneling current increases by 1 order of magnitude. ${ }^{43}$ The electric filed $E(\mathrm{~V} / \AA ̊$ ) in the tunneling junction can then be approximated with the simplified plate capacitor model according to the formula $E(I)=4 / 8$ $-\log (I / 40){ }^{44}$ Thus, the range of tunneling currents used in

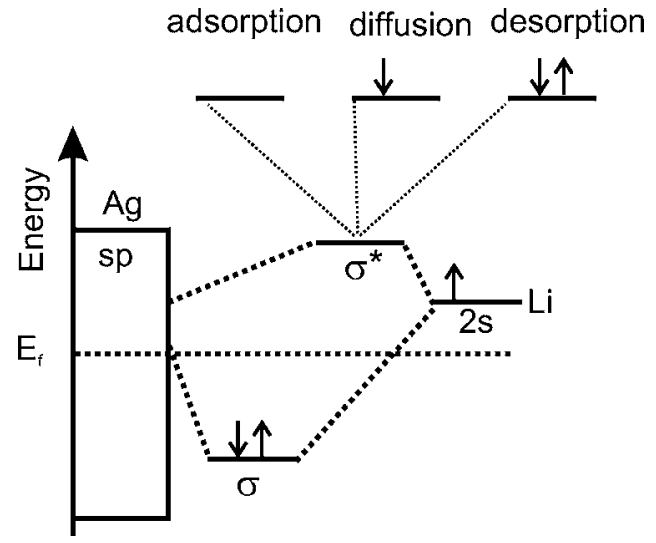

FIG. 6. Two molecular orbitals are formed upon Li adsorption on Ag. The bonding orbital is populated by two electrons (one from $\mathrm{Li}$ and one from $\mathrm{Ag}$ ) and the antibonding orbital is unoccupied. The temporary population of the antibonding orbital with one electron induces the diffusion, while the population of the antibonding orbital with two electrons might cause the desorption of the Li atom.

the experiment stimulates a large variation of the electric field between the tip and the sample and the hopping yield for a particular manipulation voltage should vary with the current for a field driven mechanism. Figure 5(c) shows the dependence of the manipulation yield for a manipulation voltage of $3.5 \mathrm{eV}$ and different manipulation currents (50, $100,150,200$, and $250 \mathrm{pA}$ ). It is obvious that the manipulation yield does not depend on the current. Thus, we can exclude a field effect as the primary hopping mechanism.

As a possible reaction mechanism, we propose electron transfer from the STM tip into an unoccupied state induced by the single $\mathrm{Li}$ atom. This is in line with our spectroscopic measurements, which show the onset of a Li-induced state at $3.6 \mathrm{eV}$. This state lies, therefore, near the threshold energy for the induced diffusion.

The mechanism of the induced diffusion can now be explained by a Newns-Anderson model, which is an important model to describe the electronic structure of a simple chemisorption system. ${ }^{45,46}$ In this model, the valence $s$ level of an alkali-metal atom hybridizes with the empty $s p$ level of a substrate when approaching the metal and forms two states, a bonding and an antibonding one. ${ }^{47}$ Thus, the single Li $2 s$ splits via bond formation with $\mathrm{Ag}$ into bonding and antibonding orbitals upon adsorption (Fig. 6). The bonding orbital is $\mathrm{Ag}$ dominated and full, while the antibonding orbital is $\mathrm{Li}$ dominated and remains empty. Upon the manipulation, one electron is transferred into the antibonding orbital. Transient population of this orbital weakens the $\mathrm{Li}-\mathrm{Ag}$ bond and induces the diffusion of the $\mathrm{Li}$ atom. The double occupation of the antibonding orbital would probably result in the desorption of the $\mathrm{Li}$ atom as the bond strength decreases additionally (Fig. 6). A similar explanation was already used to explain the breaking of chemical bonds in different electroninduced reactions. It has been shown that hydrogen desorption from hydrogen terminated silicon (100) surfaces occurs due to the temporary population of the $\sigma^{*}(\mathrm{H}-\mathrm{Si})$ orbital. ${ }^{48}$ Furthermore, the breaking of the $\mathrm{C}-\mathrm{Si}$ covalent bond between benzene and $\mathrm{Si}(100)$ was induced via transient 
ionization of a $\pi$ state of the molecule resulting in desorption of benzene. ${ }^{49}$ Also, the dissociation of molecules such as $\mathrm{O}_{2}$ and benzene was initiated by electronic transitions involving the unoccupied states formed by the respective $\pi$ orbitals. ${ }^{44,50}$

\section{CONCLUSIONS}

Employing low-temperature STM, we have investigated the adsorption of $\mathrm{Li}$ adatoms on $\mathrm{Ag}(001)$. $\mathrm{Li}$ adsorption is associated with the formation of pairs with one preferred adatom distance. No preferential adsorption site for Li adatoms was observed on $\operatorname{Ag}(001)$. We propose that interactions between adatoms leading to the pair formation are indirect and substrate mediated. The electron transfer from the STM tip into $\mathrm{Li}$ atoms induces the diffusion of single adatoms, an effect attributed to the temporary filling of an antibonding Li-induced electronic state.

\section{ACKNOWLEDGMENT}

M.N. is indebted to the University of Wroclaw for financial support (Grant No. 2016/W/IFD/2007).
*Electronic address: simic@ @hi-berlin.mpg.de

${ }^{1}$ G. Ertl, M. Weiss, and S. Lee, Chem. Phys. Lett. 60, 391 (1979).

${ }^{2}$ L. J. Whitman and W. Ho, J. Chem. Phys. 89, 7621 (1988).

${ }^{3}$ A. Cupolillo, G. Chiarello, V. Formoso, D. Pacile, M. Papagno, F. Veltri, E. Colavita, and L. Papagno, Phys. Rev. B 66, 233407 (2002).

${ }^{4}$ S. A. Lindgren and L. Wallden, Phys. Rev. Lett. 59, 3003 (1987).

${ }^{5}$ J. M. Carlsson and B. Hellsing, Phys. Rev. B 61, 13973 (2000).

${ }^{6}$ E. V. Chulkov, J. Kliewer, R. Berndt, V. M. Silkin, B. Hellsing, S. Crampin, and P. M. Echenique, Phys. Rev. B 68, 195422 (2003).

${ }^{7}$ I. Langmuir and K. H. Kingdon, Science 57, 58 (1923).

${ }^{8}$ I. Langmuir and K. H. Kingdon, Proc. R. Soc. London, Ser. A 107, 61 (1925).

${ }^{9}$ I. Langmuir and K. H. Kingdon, Phys. Rev. 34, 129 (1929).

${ }^{10}$ I. Langmuir and D. S. Villars, J. Am. Chem. Soc. 53, 486 (1931).

${ }^{11}$ I. Langmuir, J. Am. Chem. Soc. 54, 2798 (1932).

${ }^{12}$ J. B. Taylor and I. Langmuir, Phys. Rev. 44, 423 (1933).

${ }^{13}$ R. W. Gurney, Phys. Rev. 47, 479 (1935).

${ }^{14}$ M. Kerkar, D. Fisher, D. P. Woodruff, R. G. Jones, R. D. Diehl, C. F. McConville, and B. C. C. Cowie, J. Vac. Sci. Technol. A 10, 2148 (1992).

${ }^{15}$ A. V. de Carvalho, D. P. Woodruff, and M. Kerkar, Surf. Sci. 320, 315 (1994).

${ }^{16}$ M. Gierer, H. Over, H. Bludau, and G. Ertl, Surf. Sci. 337, 198 (1995).

${ }^{17}$ C. Stampfl and M. Scheffler, Surf. Rev. Lett. 2, 317 (1995).

${ }^{18}$ R. D. Diehl and R. McGrath, J. Phys.: Condens. Matter 9, 951 (1997).

${ }^{19}$ J. N. Andersen, E. Lundgren, R. Nyholm, and M. Qvarford, Surf. Sci. 289, 307 (1993).

${ }^{20}$ J. Neugebauer and M. Scheffler, Phys. Rev. Lett. 71, 577 (1993).

${ }^{21}$ C. Stampfl, M. Scheffler, H. Over, J. Burchhardt, M. Nielsen, D. L. Adams, and W. Moritz, Phys. Rev. Lett. 69, 1532 (1992).

${ }^{22}$ M. Kaack and D. Fick, Phys. Rev. B 51, 17902 (1995).

${ }^{23}$ M. M. Nielsen, S. V. Christensen, and D. L. Adams, Phys. Rev. B 54, 17902 (1996).

${ }^{24}$ S. Mizuno, H. Tochihara, A. Barbieri, and M. A. Van Hove, Phys. Rev. B 52, R11658 (1995).

${ }^{25}$ S. Mizuno, H. Tochihara, Y. Matsumoto, and K. Tanaka, Surf. Sci. 393, 69 (1997).

${ }^{26}$ H. D. Ebinger, H. J. Jänsch, C. Polenz, B. Polivka, W. Preyss, V. Saier, R. Veith, and D. Fick, Phys. Rev. Lett. 76, 656 (1996).

${ }^{27}$ M. Heyde, M. Kulawik, H.-P. Rust, and H.-J. Freund, Rev. Sci. Instrum. 75, 2446 (2004).
${ }^{28}$ The lateral resolution of the STM images is in the range of about $0.1 \mathrm{~nm}$.

${ }^{29}$ A. Bogicevic, Phys. Rev. Lett. 82, 5301 (1999).

${ }^{30}$ J. I. Pascual, A. Dick, M. Hansmann, H. P. Rust, J. Neugebauer, and K. Horn, Phys. Rev. Lett. 96, 046801 (2006).

${ }^{31}$ O. Jeandupeux, L. Bürgi, A. Hirstein, H. Brune, and K. Kern, Phys. Rev. B 59, 15926 (1999).

${ }^{32}$ N. W. Ashcroft and N. D. Mermin, Solid State Physics (HRW, Philadelphia, 1976).

${ }^{33}$ F. Stern, Phys. Rev. Lett. 18, 546 (1967).

${ }^{34}$ K. H. Lau and W. Kohn, Surf. Sci. 75, 69 (1978).

${ }^{35}$ J. Repp, F. Moresco, G. Meyer, K.-H. Rieder, P. Hyldgaard, and M. Persson, Phys. Rev. Lett. 85, 2981 (2000).

${ }^{36}$ N. Knorr, H. Brune, M. Epple, A. Hirstein, M. A. Schneider, and K. Kern, Phys. Rev. B 65, 115420 (2002).

${ }^{37}$ F. Silly, M. Pivetta, M. Ternes, F. Patthey, J. P. Pelz, and W.-D. Schneider, Phys. Rev. Lett. 92, 016101 (2004).

${ }^{38}$ K. Morgenstern and K.-H. Rieder, New J. Phys. 7, 139 (2005).

${ }^{39}$ The existence of a surface state on $\mathrm{Ag}(001)$ has been predicted by Kolb et al. but it was never directly observed in ultrahighvacuum conditions [D. M. Kolb, W. Boek, K. M. Ho, and S. H. Liu, Phys. Rev. Lett. 47, 1921 (1981)]. L. Savio, L. Vattuone, M. Rocca, V. De Renzi, S. Gardonio, C. Mariani, U. del Pennino, G. Cipriani, A. Dal Corso, and S. Baroni performed a combined $a b$ initio theoretical study and ultraviolet photoemission spectroscopy experiment on $\operatorname{Ag}(001)$ but they also did not provide direct evidence for the existence of a surface state [L. Savio et al., Surf. Sci. 486, 65 (2001)].

${ }^{40}$ A. Goldmann, V. Dose, and G. Borstel, Phys. Rev. B 32, 1971 (1985).

${ }^{41}$ B. C. Stipe, M. A. Rezaei, W. Ho, S. Gao, M. Persson, and B. I. Lundquist, Phys. Rev. Lett. 78, 4410 (1997).

${ }^{42}$ B. C. Stipe, M. A. Rezaei, and W. Ho, Science 279, 1907 (1998).

${ }^{43}$ J. K. Gimzewski and R. Moller, Phys. Rev. B 36, 1284 (1987).

${ }^{44}$ T. Komeda, Y. Kim, Y. Fujita, Y. Sainoo, and M. Kawai, J. Chem. Phys. 120, 5347 (2003).

${ }^{45}$ P. W. Anderson, Phys. Rev. 124, 41 (1961).

${ }^{46}$ D. M. Newns, Phys. Rev. 178, 1123 (1969).

${ }^{47}$ B. N. J. Persson and H. Ishida, Phys. Rev. B 42, 3171 (1990).

${ }^{48}$ T.-C. Shen, R. Rousseau, S. N. Patitsas, G. P. Lopinski, R. A. Wolkow, and T. Seideman, Science 268, 1590 (1995).

${ }^{49}$ S. Alavi, R. Rousseau, S. N. Patitsas, G. P. Lopinski, R. A. Wolkow, and T. Seidman, Phys. Rev. Lett. 85, 5372 (2000).

${ }^{50}$ W. Ho, J. Chem. Phys. 117, 11033 (2002). 